\title{
Study of Cu (Ii) Removal using Chemically Modified and Raw Organic Adsorbent
}

\author{
${ }^{1 *}$ Yugan Chandaran, ${ }^{1}$ Nazlina Bt Zulbadli \\ ${ }^{1}$ Faculty of Engineering and the Built Environment, SEGi University. \\ *Yupac94@gmail.com
}

\begin{abstract}
Wastewater problem is increasing lately due to uncontrollable disposals from industries and poor wastewater management. Wastewater discharged from industries contains dyes, heavy metal compounds, oil, etc and these contaminants will contaminant drinking water. Consuming water which is polluted by heavy metals such as copper, may result to human health problems, like anaemia, mental disorder, damages internally, nausea etc. The objective of this research is to study the effectiveness of copper, $\mathrm{Cu}$ (II) removal using organic adsorbent under the effect of varying $\mathrm{pH}$ and dosage of adsorbent. For this reason, watermelon rind was chosen as an organic adsorbent to remove $\mathrm{Cu}$ (II) from synthetic wastewater. Watermelon rind was chemically modified with hydrochloric acid to enhance the removal efficiency and the adsorption performance was compared with untreated organic adsorbent. It was found that the optimum $\mathrm{pH}$ for $\mathrm{Cu}$ (II) is 7. Adsorption equilibrium was described using Langmuir isotherm model and showed that the modified watermelon rind has a better loading capacity of metal ions compared to the unmodified watermelon rind. FTIR analysis shows that the peak shifts under carbonyl of unmodified watermelon rind differs from modified watermelon rind, due to the existing level of energy naturally in unmodified watermelon rind of copper to bind with carboxylic acid groups. The FESEM image study of modified watermelon rind shows that the structures are in irregular shapes and rough, tend to adsorb more due to higher porosity. Results show that the removal efficiency of modified watermelon rind was found to be higher (79.07\%) as compared to raw watermelon rind $(58.97 \%)$. It shows that acid modified watermelon rind has improved the removal efficiency of the adsorbent.
\end{abstract}


Keywords: Adsorption, Organic adsorbent, Watermelon rind, Copper, Hydrochloric acid, Wastewater

\section{Introduction}

Wastewater is defined as waste discharged from multiple sources which is in a form of liquid. Wastewater management is very important to the nation in order to avoid water pollution which may create discomfort for the community and affects the domestic situation. South Africa is one of the countries that have encountered problems like high risk of waterborne diseases, insufficient of drinking water and contaminating available water source (Jonnalagada S.B. and Mhere G., 2001 and Das J. and Acharya B.C., 2003). Due to poor wastewater management and treatment, it is proven that poor wastewater management in a country can cause problems and it is a big challenge to control this global issue. To mitigate this, wastewater can be treated and reused as it benefits the environment and reduces the effects and impacts on environment. However, the cost of providing water for transport or to dispose waste water is too high and expensive. This can be reduced by treating the waste water, in various options. The treatment of waste water highly depends on the type of wastewater contaminants, such as heavy metals, waste products, microorganisms which causes problem to ecosystem (Tukura B.W. et al., 2009). Wastewater also contains certain type of materials, such as organic waste from human and food, wastes from industries, fats and oils, chemicals, metals and paint. All these types of materials contained in wastewater can cause severe health issues to human health. Heavy metals such as lead, zinc, copper, arsenic and many other heavy metals contained in wastewater and discharged from industry has caused some major pollution and environmental issues in India (Srivastara, 2006). To reduce this problem, various methods are used to remove heavy metals from waste water. Ion exchange, reverse osmosis, nano filtration, ultrafiltration, chemical precipitation, coagulation/flocculation, adsorption and many other several methods or techniques used to remove heavy metal and toxic contaminants from waste water (Fenglian and Qi W., 2011). 
Among all methods, adsorption is one of the process that widely used in industry to treat wastewater. Adsorption is the suitable and frequently used method to remove heavy metal ions from many industry effluents (Gottipati and Mishra, 2012). It is used mostly because it has a lot of attributes and advantages compared to any other methods. The main advantage is, adsorption improves in heavy metal removal efficiency and it has reduced the cost by using low cost adsorbents. Some mainly used adsorbents for the process of adsorption is activated carbon industrial solid wastes and clay minerals (Wilson et al., 2006 and Wang et al., 2008). Adsorption removes both soluble and insoluble organic pollutants up to $99.9 \%$ (Ramos et al., 2002). Adsorbents can be either organic or inorganic. Organic adsorbents are like watermelon rind, orange peel, banana peel and many types of fruit peels has shown good adsorption capacity. This is advantageous as organic adsorbents are low in cost and not harmful throughout the process as compared to inorganic adsorbents. Out of all the organic adsorbents, studies have proven that the watermelon rind has a great capability of removing many pollutants like heavy metal and dye (Demirbas A. 2008 and Akpor O.B., 2011).

Adsorbent can be modified or treated with many chemical reagents (Feng $\mathrm{N}$ et al., 2011, Khormaei M et al., 2007 and Gerola G.P et al., 2013). Chemically treated adsorbents resulted to remove heavy metals more effectively and has higher efficiency compared to the non-treated adsorbents (Yargic A.S et al., 2015). Thus, the purpose of this research is to study the effectiveness of watermelon rind as an organic adsorbent to treat copper in waste water. The used of watermelon rind as adsorbent has not been studied as extensively as other biosorbents. Parameters such as $\mathrm{pH}$ and adsorbent dosage were studied for this research. Besides, the Langmuir adsorption isotherm study was also conducted to compare the unmodified and modified adsorbent.

\section{Materials and methods}

\subsection{List of materials}

Watermelon rind was collected from fruit stall in Kota Damansara, Selangor (Malaysia). Copper (II) nitrate was purchased from $\mathrm{HmbG}$ 
Chemicals. The hydrochloric acid (37\%) was purchased from Friendemann Schmidt and sodium hydroxide was purchased from R\&M Chemicals.

\subsection{Preparing chemically modified adsorbent}

Watermelon rind was washed several times with distilled water to remove soluble impurities, and dried in an oven. Watermelon rind was dried in oven at temperature range from $60^{\circ} \mathrm{C}$ to $70^{\circ} \mathrm{C}$ (Koel B. et al., 2012, Ibrahim et al., 2016). The watermelon rind was then converted into fine powder using mechanical grinder and sieved to get the adsorbent in the range of 150 to 300 $\mu \mathrm{m}$. Then, the adsorbent was left to dry overnight at room temperature and kept in air tight container. In order to build the capacity of the adsorbent to uptake adsorbate ions, the adsorbent was soaked in $0.1 \mathrm{M}$ hydrochloric acid for 4 hours (Lakshmipathy R. and Sarada N., 2015). The chemically modified adsorbent was then filtered and dried for 48 hours at $60{ }^{\circ} \mathrm{C}$ until a constant weight was obtained and washed continuously with distilled water until a constant $\mathrm{pH}$ was obtained.

\subsection{Preparation of synthetic copper (ii) solution (adsorbate)}

Preparation of synthetic $\mathrm{Cu}$ (II) solutions was prepared by dissolving copper nitrate into distilled water (Peck L. K. and Jin F.T., 2018). Copper (II) solution was prepared by using copper (II) nitrate and distilled water. For 1000 $\mathrm{mg} / 1$ solution, $3.801 \mathrm{~g} \mathrm{Cu}\left(\mathrm{NO}_{3}\right)_{2} \cdot 3 \mathrm{H}_{2} \mathrm{O}$ was dissolved in $1000 \mathrm{ml}$ of distilled water (Koel B. et al., 2012). Copper (II) nitrate is the preferable material to use to prepare synthetic solutions of copper (II) for this research.

\subsection{Batch mode studies}

Solution of copper (II) was then added with 0.1M Hydrochloric Acid or 0.1M Sodium Hydroxide. A pH meter (Mettler Toledo) was used to record the pH level and adjusted (Koel B. et al., 2012). The chemically treated and raw adsorbent was then mixed with adsorbate, and placed in an incubator shaker. Total of $20 \mathrm{~g}$ of sorbent was mixed with $1000 \mathrm{mg} / \mathrm{l}$ of heavy metal solution in and rotated it for 40 minutes. $1.5 \mathrm{~g} / 1$ of sorbent was used into $20 \mathrm{ml}$ of adsorbate and rotated it for 120 minutes in a speed of $50 \mathrm{rpm}$ (Lakshmipathy R. and Sarada N., 2015). $0.001 \mathrm{~g} / 50 \mathrm{ml}$ of adsorbent was mixed with $50 \mathrm{ml}$ of 
adsorbate and rotated it with a speed of $150 \mathrm{rpm}$ and last total of 210 minutes (Koel B. et al., 2012). For this research, dosage is a parameter that needs to be considered; hence the dosage of the adsorbent may be different and increase gradually. The adsorbent was mixed with adsorbates of various concentrations, while the time and speed of the rotational were constant for each dosages and experiment runs which were fixed at $120 \mathrm{rpm}$ for 3 hours.

\subsection{Field emission scanning electron microscopy (FESEM)}

FESEM JSM-7800F Prime equipment was used to observe the surface structure of all bio sorbent synthesized, both treated and non-treated. The surface of the samples were examined and scanned under an electron beam while the screen showed the image on the data that was required. FESEM works with high energy range and the result of the image is in higher resolution.

\subsection{Fourier transform infrared spectroscopy (FTIS)}

FTIR Perkin Elmer Spectrum 100 equipment was used to identify the functional groups in raw material and chemically treated biosorbents. $1 \mathrm{mg}$ of watermelon rind was mixed with $99 \mathrm{mg}$ of potassium bromide and compressed for several minutes (Koel B. et al., 2012).

2.7 Atomic absorption spectroscopy (AAS)

AAS Agilent Technologies 200 Series AA equipment was used to determine the initial and final concentration of copper in the solution. The particles used had to be atomized before analysis.

\section{Results and discussion}

\subsection{Effects of $\mathrm{pH}$}

$\mathrm{PH}$ is one of the most important factor or parameters in adsorption process. Changes in the $\mathrm{pH}$ of the solutions would affect the metal ion sorption onto active sites of adsorbent due to the competitiveness which occurs between hydrogen ions and metal ions. The batch mode studies been conducted for copper (II) solutions from the range of $\mathrm{pH}$ values between 3 to 9 under different 
dosages of adsorbent with an initial concentration of $10 \mathrm{ppm}, 0.25$ liter of volume and 3 hours of rotation using incubator shaker at $120 \mathrm{rpm}$.

Figure 1 shows the removal efficiency of copper (II) using unmodified watermelon rind, at $\mathrm{pH} 3$, the removal efficiency is in the range of $10.77 \%$ to $46.11 \%$, compared to $\mathrm{pH} 5$ and $\mathrm{pH} 7$, where the removal efficiency has a higher ranges which are $16.23 \%$ to $51.61 \%$ and $27.39 \%$ to $58.97 \%$ respectively. However, at $\mathrm{pH}$, the removal efficiency is slightly lower compared to $\mathrm{pH} 7$, which are $25.11 \%$ to $56.77 \%$.

On the other hand, Figure 2 shows the removal efficiency of copper (II) using modified watermelon rind. At $\mathrm{pH} 3$, the removal rate efficiency is in the range of $19.46 \%$ to $54.77 \%$, compared to $\mathrm{pH} 5$ and $\mathrm{pH} 7$, where the removal efficiency has higher ranges which are $24.96 \%$ to $64.17 \%$ and $33.65 \%$ to $79.07 \%$ respectively. Similarly with modified watermelon rind, at $\mathrm{pH} 9$, the removal efficiency is slightly lower compared to $\mathrm{pH} 7$, which are $28.77 \%$ to $74.82 \%$.

From Figure 1 and 2, it shows that the removal efficiency of copper (II) using modified watermelon rind has a higher rate of removal compared to unmodified watermelon rind. The highest removal efficiency of copper (II)

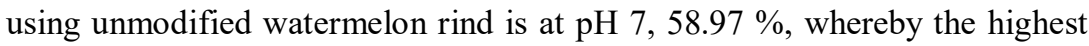
removal efficiency of copper (II) using modified watermelon rind is at $\mathrm{pH} 7$ also, $79.07 \%$. This is because further increase in $\mathrm{pH}$ to $\mathrm{pH} 9$ would result in precipitation from copper (II) to copper $(\mathrm{OH})_{2}$. The results obtained are comparable with the works from Koel B. et al. (2012). The authors found that the optimum $\mathrm{pH}$ for copper (II) sorption by watermelon rind is $\mathrm{pH} 8$. The difference of the optimum $\mathrm{pH}$ is due to the used of unmodified watermelon rind in their study.

Inversely, William J. and Wilfred L. (2018) reported that if the $\mathrm{pH}$ of the solution contained is fewer than 5.9, the surface of the watermelon rind will have high amount of positive charges of density, so it prevents from up taking copper (II) or the removal rate may be low. Similar to the works published by Koel B. et al. (2012), an increase in the level of the $\mathrm{pH}$ may influence the negatively charged surface of the watermelon rind to adsorb copper (II) ions. 
The optimum level of the $\mathrm{pH}$ was reported to be at 7.8 and anything beyond that, the removal capacity of copper (II) by watermelon rind started to drop.

\subsection{Effects of adsorbent dosage}

The experiment was also conducted using different adsorbent dosages at fixed conditions such as the initial concentration of $10 \mathrm{ppm}$, contact time of 3 hours and volume of the solution is $250 \mathrm{ml}$. The dosages differ in the range of 3, 7 and 9 grams. The results showed that the removal efficiency of copper (II) is the high at increasing dosage which is 9 grams compared to 7 grams and 3 grams. This is because an increase in the adsorbent dosage would increase the available active surface areas to absorb heavy metal ions Hence, it is expected that drastic increase have been observed in removal efficiency as the dosage increases. This effect of adsorbent dosage applies for both modified and unmodified watermelon rind batch mode studies. Figure 1 shows the removal efficiency of copper (II) using unmodified watermelon rind, the removal efficiency in 3 grams is lower than 9 grams at optimum $\mathrm{pH}$ of 7 , which are $27.39 \%$ and $58.97 \%$ respectively. On the other hand, Figure 2 shows the removal efficiency of copper (II) using modified watermelon rind, the removal efficiency in 3 grams is lower than 9 grams at optimum $\mathrm{pH}$ of 7 , which are $33.65 \%$ and $79.07 \%$. In accordance to Koel B. et al., (2012), the maximum removal rate was recorded at the dosage of $0.002 \mathrm{~g} / \mathrm{l}$ and the removal efficiency was recorded at $84 \%$. According to his investigation, the removal rate of copper (II) increases as the dosage of the watermelon rind increased, due to the amount of active areas to bind, however, the author also stated that after certain amount of dosage increased, the change in final concentration of the solution were little, was because of the amount and concentration of watermelon rind were more and tend to block the active areas from binding. 


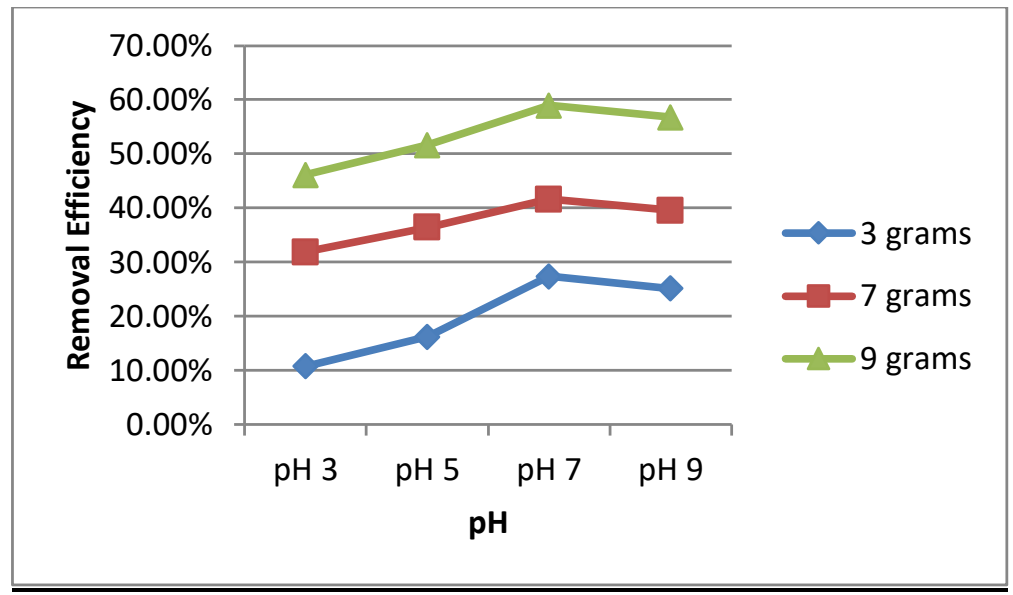

Figure 1. Removal efficiency of copper (ii) using unmodified adsorbent

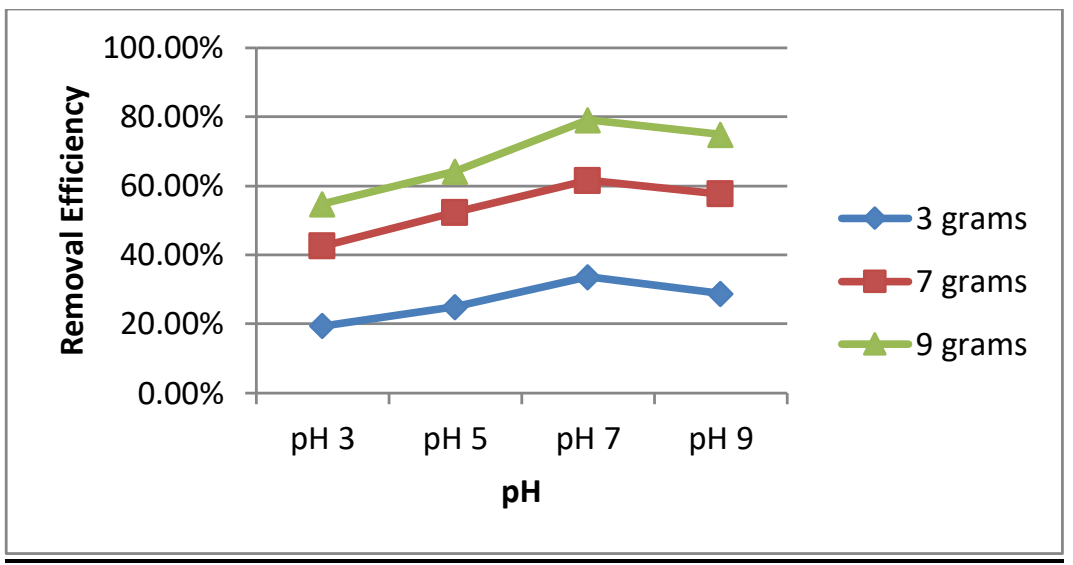

Figure 2. Removal efficiency of copper (ii) using modified adsorbent

\subsection{Effect of copper (ii) concentration on adsorption isotherm}

The experiment was then conducted to determine the maximum loading capacity of copper (II) concentration in solution and to identify the sorption equilibrium. Initial concentration varies from 10ppm, 20ppm and 30ppm, with other fixed conditions such as the volume of the solution is $250 \mathrm{ml}, 1$ hour of contact time at $120 \mathrm{rpm}$ in incubator shaker, $\mathrm{pH}$ of 5 and 10 grams of adsorbent. This study was tested for both modified and unmodified watermelon rind. The adsorption data had been fitted to a Langmuir isotherm model. This model was selected as it had been proven by many other researchers' that this model of isotherm fits the best compared to Freundlich and Temkin. The Langmuir 
model is able to explain the relationship between loading capacity of metal and heavy metal ion concentration at equilibrium state.

Based on this part of study, the Langmuir isotherm model showed that the modified watermelon rind has a better loading capacity of metal ions compared to the unmodified watermelon rind. Soaking of raw watermelon rind in $\mathrm{HCl}$ for 24 hours had increased the sorption capacity of copper (II) ions by activating the attributes of absorbing mainly because of desorption of alkaline metals and electronegativity. Figure 3 shows the Langmuir isotherm model for unmodified watermelon rind, the regression value, $\mathrm{R}^{2}$ is 0.9934 , which means the data fits into Langmuir isotherm model and shows that the watermelon rind usage as an adsorbent is suitable for copper (II) ions removal. Figure 4 shows the Langmuir isotherm model for modified watermelon rind, the regression value, $\mathrm{R}^{2}$ is 0.9922 , which means the data fits into Langmuir isotherm model and also shows that the modified watermelon rind usage as an adsorbent is suitable for copper (II) ions removal. In accordance to Koel B. et al. (2012), the regression value for his investigation was recorded at 0.9859 , and it showed that the data fits the Langmuir isotherm model and watermelon rind is good to adsorb copper (II) ions. William J. and Wilfred L. (2018) reported that the regression value of 0.87 suits well in Langmuir isotherm model and showed that watermelon rind is suitable for copper (II) ions removal.

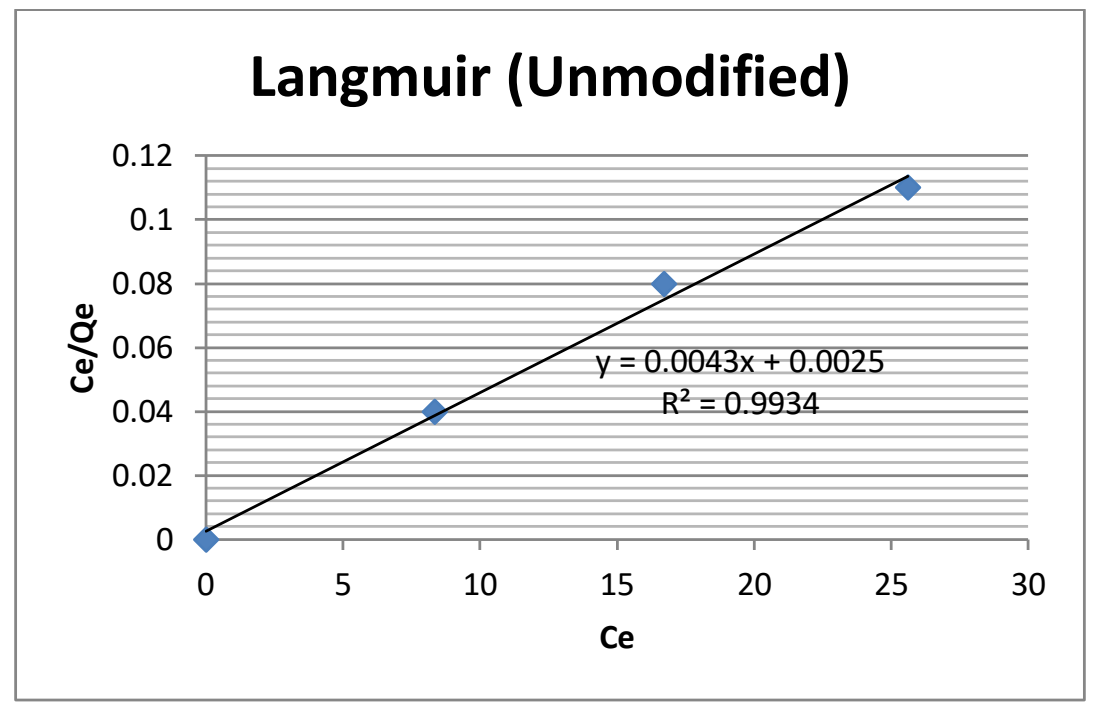


Figure 3. Langmuir isotherm model for unmodified adsorbent

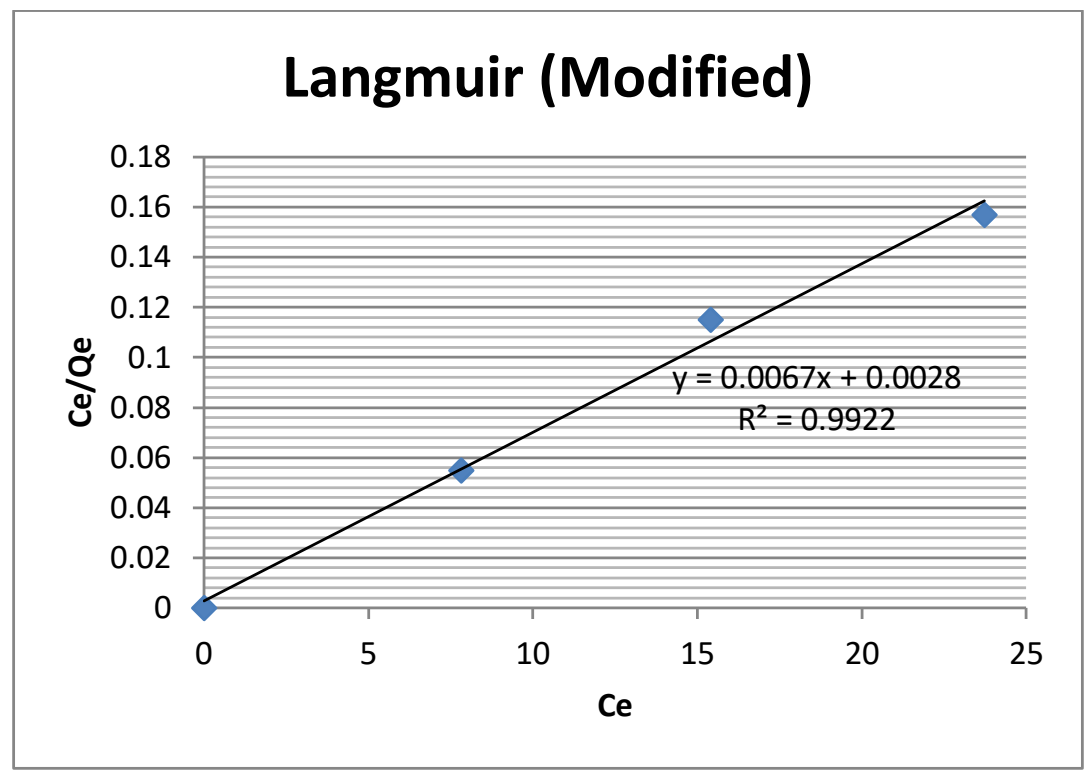

Figure 4. Langmuir isotherm model for modified adsorbent

\subsection{Fourier transform infrared spectroscopy (FTIS)}

Fourier Transform Infrared Spectroscopy (FTIS) analysis is used to determine the features of functional groups of chemically treated and nontreated watermelon rind. Referring to the spectra of Figure 5, the first peak of the unmodified watermelon rind and modified watermelon rind is recorded at $3326.66 \mathrm{~cm}^{-1}$ and $3369.12 \mathrm{~cm}^{-1}$, respectively, which relates to $-\mathrm{OH}$ stretching vibrations of the cellulose. The next peak for both unmodified and modified watermelon rind was recorded at $1602.27 \mathrm{~cm}^{-1}$ and $1647.46 \mathrm{~cm}^{-1}$, respectively, which relates to $-\mathrm{C}=\mathrm{O}$ stretching of the carboxylic acids. The $-\mathrm{COO}^{-}$ asymmetric vibrations of ionic carboxylic groups are found when the third peak and the fourth peak for both unmodified and modified watermelon rind were recorded. The difference occurred in wavelength numbers in spectra for unmodified and modified watermelon rind is because of the desorb process that took part naturally in desorbing copper ions while the protons were added to the available active sites. The shifts in wavelength numbers for modified watermelon rind have shown that the acidic groups a higher capability to adsorb heavy metals. The peak shifts under carbonyl of unmodified 
watermelon rind differs from modified watermelon rind, due to the existing level of energy naturally in unmodified watermelon rind of copper to bind with carboxylic acid groups. In accordance to Lakshmipathyand Sarada (2015), the FTIR analysis were made for raw watermelon rind, modified watermelon rind using hydrochloric Acid, lead loaded with modified watermelon rind and copper loaded with modified watermelon rind. The results of wave numbers recorded by the researchers are almost similar to this study.

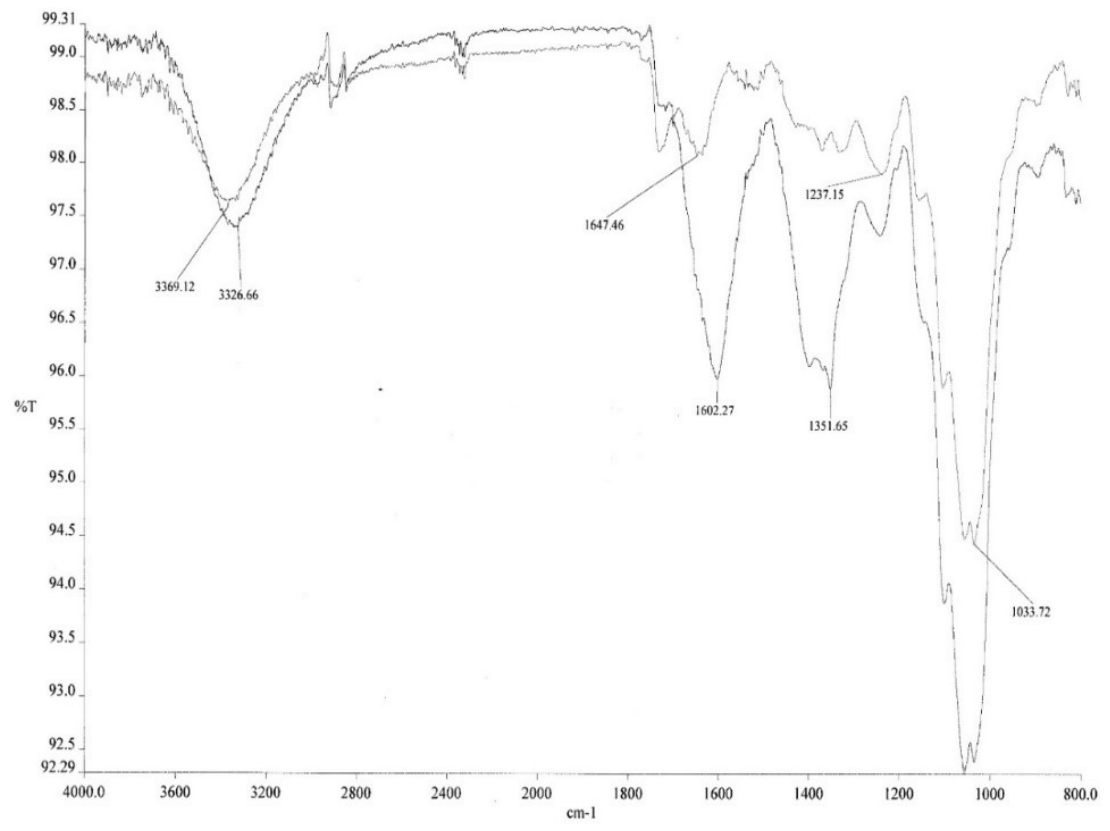

Figure 5. FTIS spectra of a) modified and b) unmodified adsorbents

\subsection{Field emission scanning electron microscope (FESEM)}

In this characterization study, both unmodified and modified watermelon rind was examined and characterized. Observation was made on the upgraded capability of the adsorption implementation of removing copper (II) ions. Main observation made is on the modified watermelon rind to investigate the specific transformation on the formed surface. Figure 6 (a) - (d) shows the FESEM images of unmodified watermelon rind. Figure 7 (a) - (d) shows the FESEM images of modified watermelon rind. 


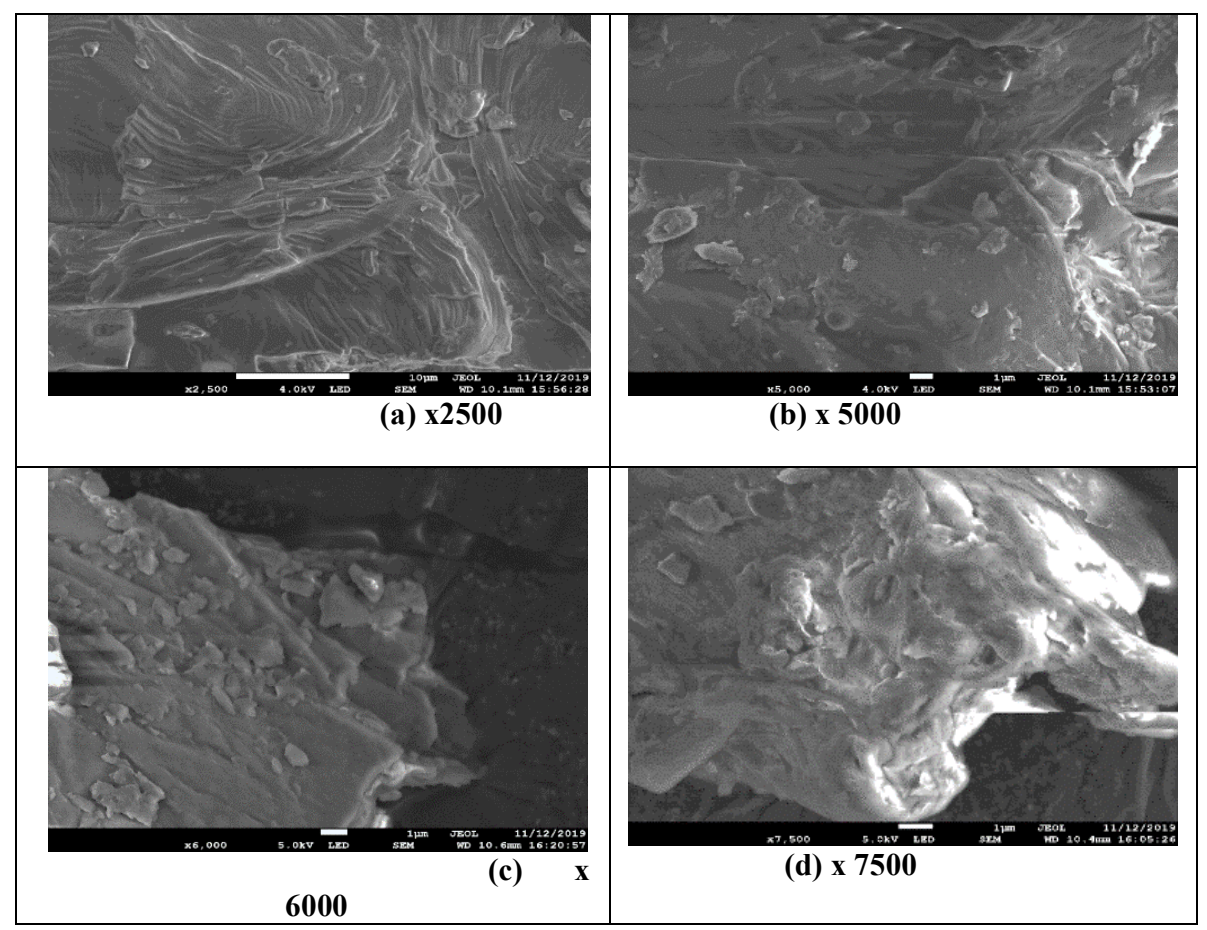

Figure 6. FESEM images of unmodified adsorbents

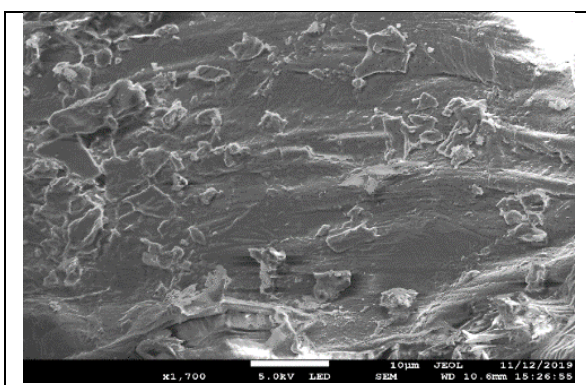

(a) $\times 1700$

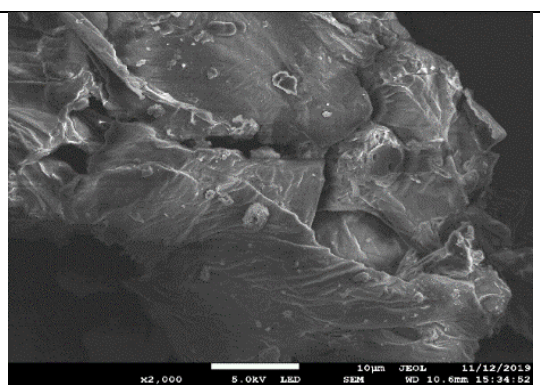

(b) $\times 2000$ 


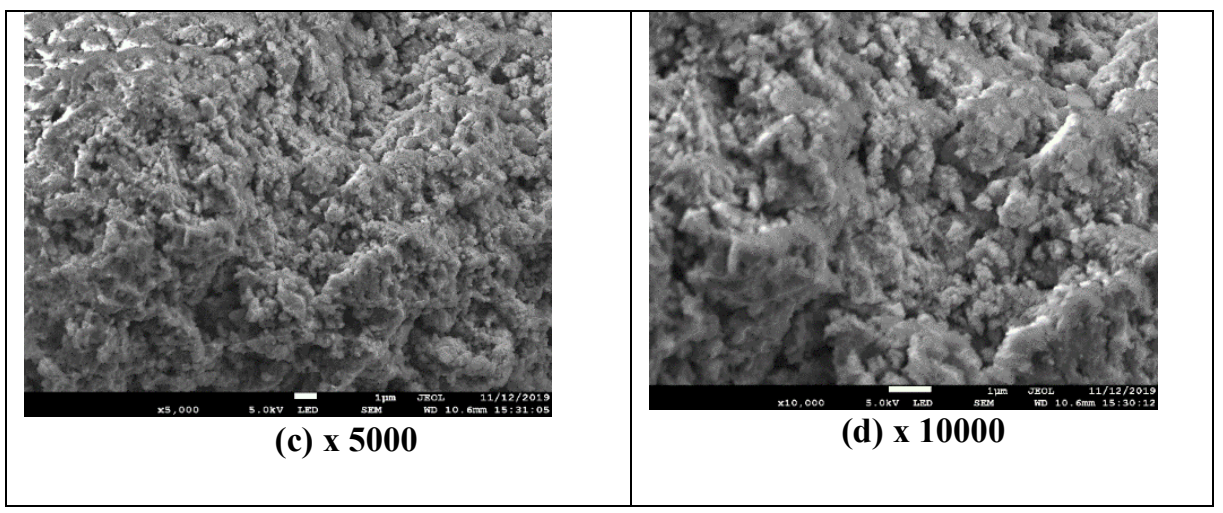

Figure 7. FESEM images of modified adsorbents

In comparison of Figure 6 and Figure 7, the image shows that there are clear transformations and adjustments on the surfaces. On the surfaces of unmodified watermelon rind, it is observed that the fibers found are fine and smooth. On the other hand, the image of modified watermelon rind as shown in Figure 7, it is observed that the surface of the structures are in irregular shapes and rough The irregular surface structure for the modified watermelon rind enables a better adsorption capacity, due to the high porosity level formed and an increase in surface area for binding which leads to a higher level of capacity to adsorb heavy metal, copper (II) ions for this study compared to unmodified watermelon rind. In accordance to Peck L.K. and Jin F.T. (2018), the chemically treated watermelon rind with citric acid and sulphuric acid has more porosity level and has a larger surface area compared to raw watermelon rind. Ibrahim et al. (2016) reported that the improved surface area and porosity level of watermelon rind after chemically treated is because of mortification of the lignin and hemicellulose components in watermelon rind.

\section{Conclusion}

As a conclusion, this study of adsorption of copper (II) ions using raw organic adsorbent and chemically treated organic adsorbent using hydrochloric acid, which the organic adsorbent is the watermelon rind, has proven as a good adsorbent to remove copper (II) ions. The optimum $\mathrm{pH}$ is recorded at $\mathrm{pH}$ of 7 and the optimum dosage of adsorbent is recorded at 9 grams of adsorbent. Adsorption isotherm model for this study has fitted into Langmuir isotherm 
model, shows that the watermelon rind is a good adsorbent and suitable adsorbent for adsorption usages, particularly for adsorbing copper (II) ions. As the regression values obtained for both modified and unmodified watermelon rind is 0.9922 and 0.9934 , respectively. FTIR characterization has confirmed that the modification of watermelon rind and raw watermelon rind has certain types of functional groups, which can uptake metal ions; it was observed from the spectra of the wave numbers for the samples prepared. FESEM analysis has shown the adsorbent image before and after being chemically treated, the modified watermelon rind has more porosity level and has a bigger surface area to bind.

\section{Acknowledgement}

The authors would like to thank SEGi University for the research and financial assistance given.

\section{References}

Akpor, O. B. "Wastewater effluent discharge" 3rd International Conference on Chemical and Environmental Engineering 20 (2011): 85-91.

Das J. and Acharya B.C (2003). Hydrology and assessment of lotic water quality in Cuttack City, India. Water, Air and Soil Pollution. 150: 163 175 .

Demirbas A. (2008). Heavy metal adsorption onto agro based waste materials: A review. Journal of Hazardous Material. 157: 220 - 229.

Feng N, Guo X, Liang S, Zhu Y. and Liu J. (2011). Biosorption of heavy metals from aqueous solutions by chemically modified orange peel. Journal of Hazardous Materials. 185: 49 - 54

Fenglian F. and Qi W. (2011).Removal of Heavy Metal ions from wastewater: A review. Journal of Environmental Management. 92 (3): 407 - 418.

Gerola G.P, Boas, G. and Dragenski D.C. (2013). Utilization of passion fruit skin by product as lead (II) ion biosorbent. Water, Air and Soil Pollution. 224: 1446

Gottipati R. and Mishra S. (2012). Application of response surface methodology for optimization of $\mathrm{Cr}$ (III) and $\mathrm{Cr}$ (VI) adsorption on commercial activated carboms. Research Journal of Chemical Sciences. 2 (2): $40-48$. 
Ibrahim, A., Yusof, N., Lee, P. Y., Lee, R. N. S. and Zahrim, A. Y. "Adsorption study of Ammonia Nitrogen by watermelon rind," Earth and Environmental Science 36 (2016): 012020.

Jonnalagada S.B. and Mhere G. (2001). Water quality of Odzi River in the eastern highlands of Zimbabwe. Water Resources. 35: 2371 - 2376.

Khormaei M., Nasernejad B. and Eslamzaden T. (2007). Copper biosorption from aqueous solutions by sour orange residue. Journal of hazardous Materials. 149: $269-274$.

Koel B., Gandhimathi R., Nidheesh P.V. and Bharathi (2012). A novel Agricultural Waste Adsorbent, Watermelon Rind for the Removal of Copper. Iranica Journal of Energy and Environment 3(2): 143 - 156, 2012.

Lakshmipathy R. and Sarada N.C. (2015).Metal ion free watermelon rind as adsorbent for removal of Copper ions. Desalination and Water Treatment (2015): $1-11$

Peck L.K. and Jin F.T. (2018). Screening of Significant Parameters affecting Zinc (II) Adsorption by chemically treated Watermelon Rind. Progress in Energy and Environmental. Vol 6: 19 - 32.

Quek S.Y, Chok N.K. and Swedland P. (2007). The physiochemical preparation of spray dried watermelon powder. Chemical Engineering Processes. 46: 386 - 392.

Ramos R. L., Jacome L.A.B, Barron J, Rabio and Coronado (2002). Adsorption of Zinc (II) from an aqueous solution onto activated carbon. Journal of Hazardous Materials. 90: 27 - 38.

Srivastara (2006). Novel Bio filtration methods for the treatment of heavy metals from industrial waste water. Journal Hazardous 151: $1-8$.

Tukura B.W., Kagbu J.A. and Gimba C.E. (2009). Effects of pH and Seasonal variations on dissolved and suspended Heavy Metals in Dam surface water. Chemistry Class Journal. 6: $27-30$.

Wang S, Ang H. and Tade M. (2008). Novel Applications of Red Mud as coagulant, adsorbent and catalyst for environmentally benign processes. Chemosphere. $72: 1621-1635$

William J.M. and Wilfred M.L. (2018). Assessments of Effectiveness of Watermelon Rinds on removing Copper (II) ions from synthesized. Environmental Monitoring and Protection. Vol 5, No. 3, 2018 pp. 40 - 51. 
Wilson K., Yeng H., Seo C.W. and Marshall (2006). Metal adsorption by activated carbon made from peanut shells. Bioresource Technology. 97: $2266-2270$.

Yargic A.S. , Ozbay N. , Yarbay and Onal E. (2015). Assessment of toxic copper (II) biosorption from aqueous solution by chemically treated tomato waste. Journal of Cleaner Production. 88: 152 - 159 